\section{Confiabilidade das informações obtidas de informante secundário em inquéritos de saúde}

\author{
Reliability of information from proxy \\ respondents in health surveys
}

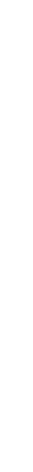

Abstract

The use of an be a source of information bias in epidemioogical studies. This study assesses the agreement eliability, magnitude, and direction of possible biases as well as the influence of proxy characeristics on the disagreements between self-report and proxy reports among 710 pairs of primarysecondary respondents. The influence of the proxy perspective on pair-agreement was also assessed, y compa health a use. In general, the results showed good agree ment and reliability. Disagreements were statistically associated with the following characteristics of proxy respondents: older age, lower schooling, and the proxy's knowledge of the primary respondent's health. Adults agreed more in evaluating the health of elderly when they used the elderly primary informant's perspective to respond. The results confirm the presence of bias when proxy respondents are used for hypertension and medical consultations.

Bias (Epidemiology); Health of the Elderly; Health Evaluation; Reproducibility of Result
Renata Jardim 1,2

Sandhi Maria Barreto 1 Luana Giatti 1

\section{Introdução}

Erros na medidac res fontes de viés em os quais podem levar a conclusões espúrias soore a relação entre a exposição e a doença. Do ser definidos como a diferença entre a medida da exposição e a exposição verdadeira 1 . Nesse risma, o uso do informante secundário pode ser uma importante fonte de viés na mensuração de

Os informantes secundários sã studos epidemiológicos quando a população estudada, por alguma razão (morte, demência, debilidade ica, desconhecimento), é incapaz indivíduos avaliados em um es ssim maior representatividade ao grupo esturitos popude cundários, alguns estudos têm mostrado que a concordância entre o indivíduo e seu informante é melhor para os componentes físicos e pior relacionados a qualidade de vida 4,56. Os indivíduos que fornecem a inforr sado tendem a subestimar ou superestimar os escores de qualidade de vida do informante primário 7 . Similarmente, Fayers \& Machin (2000, 
apud Milne et al. 4) acreditam que informantes secundários subestimam o impacto dos aspectos psicológicos e enfatizam a importância dos sintomas físicos mais objetivos. Há evidência na literatura de que o nível de concordância entre o indivíduo e seu informante é, em algum grau, dependente da concretização, visibilidade e saliência do domínio da qualidade de vida que está sendo avaliado 4 .

A análise dos dados de um inquérito de saúde realizado em 1998 pares idoso-informante secundário 7 mostrou que a completude da informação secundária é fortemente influenciada pela natureza da questão e pelo relacionamento do informante com o idoso, co-residência e tempo que o informante conhece o idoso. A concordância pelo kappa foi fortemente dependente da pergunta, um pouco menos da co-residência e, sofreu pequena influência do grau de relacionamento entre o informante secundário e o idoso.

Armstrong et al. 1 concluíram em revisão sobre o tema que a concordância entre a informação do indivíduo e de seus informantes secundários varia muito de uma exposição para outra, havendo indicações de uma boa concordância para peso, altura e nível educacional, moderada sobre tabagismo e fraca para dieta.

As diferenças encontradas nos níveis de concordância entre os pares primário-secundário nos estudos sobre qualidade de vida relacionada à saúde também podem ser justificadas pela inconsistência na perspectiva preponderante do informante secundário ${ }^{8}$. A resposta do informante secundário pode ser obtida sob duas perspectivas: a do informante primário e a sua própria. A perspectiva do informante primário é a resposta baseada em como o informante secundário pensa que o informante primário se avalia e, a perspectiva do informante secundário baseia-se em como ele próprio avalia a saúde do informante primário ${ }^{8}$. A primeira perspectiva permite avaliar a habilidade do informante secundário em compreender o ponto de vista do informante primário quando comparada com a auto-avaliação. Já a perspectiva do informante secundário expande a visão fornecida pelo informante primário, o que pode ser útil, por exemplo, em estudos sobre qualidade de vida de indivíduos com doenças crônicas. Os inquéritos populacionais periódicos permitem conhecer o perfil de saúde e a distribuição dos fatores de risco em uma população e realizar comparações temporais e espaciais ${ }^{9}$. No Brasil, a Pesquisa $\mathrm{Na}$ cional por Amostra de Domicílios (PNAD), realizada anualmente pelo Instituto Brasileiro de Geografia e Estatística (IBGE), é um inquérito de base populacional, desenvolvido com o objetivo de obter informações sobre característi- cas demográficas, habitacionais, educacionais, de trabalho e rendimentos. Nos anos de 1998, 2003 e 2008, além dessas, foram investigados diversos aspectos referentes à saúde. A PNAD prevê em sua metodologia o uso do informante secundário 9.

Existem inúmeros trabalhos importantes em saúde que utilizam os bancos de dados da PNAD 10,11,12,13, como a descrição da situação de saúde da população idosa brasileira que usou dados da PNAD 1998 13. Neste estudo, 38\% das entrevistas foram respondidas por outra pessoa, incluindo as informações sobre a percepção da própria saúde, e esta proporção diferiu entre os sexos e entre as diversas faixas etárias. Como a percepção da saúde refere-se a um julgamento subjetivo, a rigor ela não poderia ser determinada por outra pessoa. Não se sabe como a utilização de outro respondente influenciou a mensuração da avaliação da saúde e demais informações de saúde na população estudada.

Na PNAD realizada em 2003, 64\% das entrevistas dos adultos (idades superiores a 17 anos) foram respondidas por informantes secundários nas regiões metropolitanas. Entre essas entrevistas, $59 \%$ dos adultos (18-59 anos) e $41 \%$ dos idosos (60 anos ou mais) tiveram seus questionários respondidos por outros informantes que não os indivíduos pesquisados. A PNAD 2008 incluiu no suplemento de saúde perguntas sobre modos de vida. Entretanto, persiste o desconhecimento sobre os possíveis vieses de informação relacionados à utilização do informante secundário.

O presente estudo pretende contribuir para preencher essa lacuna estimando a confiabilidade entre pares indivíduo-informante secundário com relação a diversos componentes de saúde, utilizando perguntas iguais ou similares à PNAD, buscando, assim, contribuir para caracterizar um possível informante secundário ideal. As hipóteses a serem testadas são de que informantes secundários do sexo feminino, mais velhas, com maior escolaridade, que referem conhecer bem a saúde do informante primário e são esposas ou vivem com o informante primário apresentam maior concordância em suas respostas com o informante primário.

\section{Material e métodos}

Este estudo faz parte do projeto Envelhecimento e Saúde, um inquérito de base populacional cujo objetivo geral é conhecer o perfil de saúde dos idosos residentes na área de abrangência do Centro de Saúde Vila Pinho, em Belo Horizonte, Minas Gerais, e, um dos objetivos específicos, é investigar a confiabilidade de informações obti- 
das de respondentes secundários em inquéritos de saúde.

Os termos informante substituto, secundário ou proxy são sinônimos e definidos como pessoas que fornecem informações sobre a exposição ou eventos relacionados à saúde no lugar do próprio indivíduo. Neste estudo, optamos pelo termo informante secundário.

\section{População e procedimentos do inquérito}

A amostra deste inquérito baseou-se no cadastro do centro de saúde, realizado no ano de 2006 e atualizado pelos agentes comunitários de saúde (ACS). Os idosos (idade igual ou superior a 60 anos) foram selecionados por meio de amostra aleatória estratificada por sexo e idade. O cálculo do tamanho da amostra para o estudo transversal considerou prevalência de 50\%, intervalo de $95 \%$ de confiança (IC95\%), $4 \%$ de precisão e $20 \%$ de perda, totalizando 405 indivíduos estratificados por faixa etária e sexo $(69 \%$ dos idosos cadastrados). O cálculo amostral do estudo de concordância ( $n=250)$ foi baseado no coeficiente kappa mínimo de 0,6, com precisão igual a 0,10 e IC95\% para uma prevalência estimada de $50 \%$.

A coleta de dados ocorreu entre abril e outubro de 2007, sendo as entrevistas realizadas no centro de saúde, no domicílio ou no trabalho do entrevistado, previamente agendadas pelos ACS ou pelas próprias pesquisadoras.

Os instrumentos e procedimentos da pesquisa foram testados no estudo piloto em 30 idosos e em oito adultos co-residentes dos idosos. Todos os participantes do estudo assinaram o Termo de Consentimento Livre e Esclarecido.

As entrevistas estruturadas foram realizadas por entrevistadoras previamente treinadas. A confiabilidade da entrevista foi determinada em $7 \%$ dos participantes, reaplicando-se perguntas do questionário geral. Todos os questionários digitados foram conferidos manualmente. Maiores informações sobre a metodologia do inquérito estão disponíveis em Carvalho 14.

\section{População e procedimentos do presente estudo de confiabilidade}

Com base na listagem dos 405 idosos sorteados para o projeto Envelhecimento e Saúde, todos os que tinham no mínimo dois adultos co-residentes foram incluídos na amostra do presente estudo. É importante salientar que entre os adultos co-residentes existem idosos, visto que a definição de adulto utilizada foi de indivíduos com idades superiores a 17 anos.

$\mathrm{O}$ adulto $\mathrm{A} 1$ era o primeiro co-residente do idoso a responder o questionário e o adulto A2 o segundo entrevistado. O idoso respondia sobre sua saúde e depois sobre a saúde do adulto Al. O Adulto A1, além de falar sobre sua saúde, falava sobre o idoso e, o adulto A2 respondia sobre a saúde do adulto A1. Para evitar perda de informações, o informante secundário era orientado a escolher as opções de resposta que ele considerava mais adequadas baseado em seu próprio ponto de vista.

Para evitar contaminação da informação, ou seja, que um informante alterasse suas respostas por ouvir as respostas do outro, os informantes primários e secundários foram entrevistados separadamente 15 . O intervalo de tempo entre as entrevistas dos informantes primário-secundários foi de até sete dias em $91 \%$ das entrevistas entre os idosos e adultos Al e em $90 \%$ das entrevistas entre os adultos A1 e A2, sendo que $67 \%$ das entrevistas foram feitas no mesmo dia. A variável intervalo de tempo entre as entrevistas não teve associação significativa com nenhum dos desfechos analisados.

O questionário estruturado continha 50 perguntas similares ou iguais às da PNAD 2003, além de perguntas sobre tabagismo, atividade física, dieta e outras questões não analisadas neste artigo.

A concordância entre as seguintes informações foram investigadas: tabagismo durante a vida; avaliação da saúde (muito boa, boa, regular, ruim e muito ruim); relato de diagnóstico médico de hipertensão (sim/não); e consulta médica nos últimos 12 meses (sim/não). A influência no grau de confiabilidade das seguintes características do informante secundário foram testadas: grau de conhecimento da saúde do informante primário pelo informante secundário (muito bom, bom, relativamente bom, pouco ou muito pouco); parentesco (pais/filhos, cônjuge, irmãos, outros); sexo (masculino, feminino); escolaridade (0-3 anos de estudo, 4-7 anos, 8 ou mais anos de estudo); faixa etária (entre adultos: 18-39 anos, 40-59, 60 ou mais anos; entre idosos: $60-64$ anos, 65-69, 70-74, 75-79 anos e 80 anos ou mais), além do intervalo de tempo entre as entrevistas $(\leq 7$ dias, $>7$ dias).

A avaliação da saúde foi mensurada pelas duas perspectivas do informante secundário. Primeiro, esse respondia o que achava da saúde do informante primário (perspectiva do informante secundário): "De um modo geral, você considera o estado de saúde do(a) Sr(a) como: muito bom, bom, regular, ruim ou muito ruim", e depois respondia como ele achava que o informante primário via sua própria saúde (perspectiva do informante primário): "De um modo geral, o(a) Sr(a) considera o estado de saúde dele(a) como: muito bom, bom, regular, ruim ou muito ruim". 
Para descrição da freqüência, a avaliação da saúde foi agrupada em muito boa/boa/regular e ruim/muito ruim.

As variáveis explicativas testadas referentes às características dos informantes secundários que poderiam influenciar a discordância entre os pares primário-secundário foram: sexo, idade, escolaridade, grau de parentesco do informante secundário, e avaliação do mesmo sobre seu conhecimento acerca da saúde do informante primário.

\section{Análise estatística}

Utilizou-se o programa EpiData 3.1 (EpiData Association, Odense, Dinamarca) para entrada dos dados e o programa Stata (Stata Corp., College Station, Estados Unidos) para análises estatísticas. A confiabilidade entre os pares idoso-adulto, adulto-idoso e adulto-adulto foi aferida pelo Índice kappa para cada subconjunto de questões (modos de vida e avaliação da saúde, morbidade referida e uso de serviços de saúde) separadamente. A concordância mensurada pelo kappa foi classificada conforme Sneewn et al. 16, considerando índices kappa acima de 0,60 como satisfatórios e valores inferiores inaceitáveis. Os escores utilizados no kappa ponderado para a variável avaliação da saúde foram: $1 ; 0,9375 ; 0,75$; 0,4375 e 0, sendo dados por:

$$
1-\{(\mathrm{i}-\mathrm{j}) /(\mathrm{k}-1)\}^{2}
$$

em que $i$ e $j$ correspondem às linhas e colunas de cada informante e $k$ é o número máximo de possibilidades de respostas.

A seguir, analisou-se o kappa ajustado pela prevalência e a homegeneidade entre as probabilidades marginais. O kappa ajustado pela prevalência avalia a proporção de concordância entre as variáveis menos a proporção de desacordos, com o objetivo de minimizar o viés introduzido no índice kappa pela prevalência dos eventos investigados, valorizando a concordância observada. O cálculo desse kappa pode ser expresso por $2 \mathrm{Po}-1$ ou $\mathrm{Po}-(1-\mathrm{Po})$, em que $\mathrm{Po}$ é a proporção da concordância observada 17. A análise da homogeneidade entre as probabilidades marginais permite inferir se o coeficiente kappa é uma boa estimativa de confiabilidade para as variáveis selecionadas, uma vez que probabilidades marginais similares entre os dois observadores (neste caso, entre o par primário-secundário) resultam em estimativas muito próximas entre o coeficiente kappa e outras medidas de confiabilidade (Scott's PI, modelo estatístico log-linear e kappa intraclasse) 18 .

Para analisar a presença, magnitude e direção de vieses de mensuração, utilizou-se um indica- dor de viés proporcional (VP) para cada questão investigada por meio da seguinte fórmula:

$$
V P=\left(P_{p x}-P_{p r}\right) / P_{p x}
$$

em que $P_{p x}$ é a proporção de respostas positivas dadas pelos informantes primários e $P_{p r}$ a proporção de respostas positivas de acordo com os informantes secundários. Valores negativos representam superestimação, e os positivos, subestimação das respostas dos informantes secundários em comparação com as do informante primário 19 .

Finalmente, a influência de características selecionadas do informante secundário na discordância entre as informações fornecidas pelos pares foi investigada quando a concordância observada foi menor que $90 \%$. A associação dessas características com a discordância foi estimada utilizando-se o modelo de Poisson com estimação robusta da variância do erro 20 .

\section{Aspectos éticos}

O projeto desta pesquisa cumpriu os princípios éticos expressos na Declaração de Helsinki e foi aprovado pelo Comitê de Ética em Pesquisa da Universidade Federal de Minas Gerais em outubro de 2006 (parecer no ${ }^{\circ}$ 379/2006), e pelo Comitê de Ética em Pesquisa da Secretaria Municipal de Saúde de Belo Horizonte em janeiro de 2007 (parecer $n^{\circ}$. 065/2007).

\section{Resultados}

Participaram deste estudo 710 pares de informantes primário-secundários: 239 idosos-adultos, 239 adultos-idosos e 232 adultos-adultos. A média e a mediana da idade dos idosos sorteados foi de 70 e 67 anos, dos adultos A1 (entrevistados sobre sua própria saúde e a do idoso) foi de 43 e 44 anos e dos adultos A2 (entrevistados sobre sua própria saúde e a do adulto Al) foi igual a 37 e 33 anos, respectivamente. O percentual de indivíduos com 60 ou mais anos entre os adultos Al foi de $13 \%$ e entre os adultos A2 foi de $9 \%$.

A média e mediana da escolaridade foi de três anos de estudo para os idosos e de sete e oito anos de estudo para os adultos A1 e A2, respectivamente. A maioria dos informantes secundários era do sexo feminino, correspondendo a $58 \%$ dos adultos A2 e dos idosos, e $73 \%$ dos adultos A1. Os adultos A1 e A2 diferiram significativamente em relação ao sexo e idade, sendo mais freqüentes as mulheres entre os adultos Al e os jovens entre os adultos A2. Os adultos A2 tiveram a mesma distribuição de sexo dos idosos, porém, estes tinham menor escolaridade (Tabela 1). 
Distribuição relativa e absoluta de algumas variáveis dos informantes, grau de parentesco e grau de conhecimento da saúde do informante primário pelo informante secundário.

\begin{tabular}{|c|c|c|c|c|c|c|}
\hline \multirow[t]{2}{*}{ Variáveis } & \multicolumn{2}{|c|}{ Idosos } & \multicolumn{2}{|c|}{ Adultos A1 } & \multicolumn{2}{|c|}{ Adultos A2 } \\
\hline & $\mathrm{n}$ & $\%$ & $\mathrm{n}$ & $\%$ & $\mathrm{n}$ & $\%$ \\
\hline \multicolumn{7}{|l|}{ Sexo } \\
\hline Feminino & 138 & 58 & 174 & 73 & 134 & 58 \\
\hline Masculino & 101 & 42 & 65 & 27 & 98 & 42 \\
\hline \multicolumn{7}{|l|}{ Idade (anos) } \\
\hline $18-39$ & 0 & 0 & 103 & 43 & 152 & 66 \\
\hline $40-59$ & 0 & 0 & 108 & 45 & 59 & 25 \\
\hline $60-64$ & 86 & 36 & 13 & 5 & 8 & 4 \\
\hline $65-69$ & 56 & 24 & 9 & 4 & 3 & 1 \\
\hline $70-74$ & 32 & 13 & 5 & 2 & 8 & 3 \\
\hline $75-79$ & 34 & 14 & 2 & 1 & 2 & 1 \\
\hline $80-89$ & 31 & 13 & 0 & 0 & 0 & 0 \\
\hline \multicolumn{7}{|l|}{ Escolaridade (anos) } \\
\hline $0-3$ & 140 & 60 & 99 & 41 & 132 & 58 \\
\hline $4-7$ & 83 & 35 & 90 & 38 & 56 & 24 \\
\hline $8-20$ & 11 & 5 & 50 & 21 & 41 & 18 \\
\hline \multicolumn{7}{|l|}{ Parentesco } \\
\hline Filhos/Pais & 135 & 56 & 135 & 56 & 97 & 42 \\
\hline Cônjuge & 57 & 24 & 57 & 24 & 45 & 19 \\
\hline Irmãos & 3 & 1 & 3 & 1 & 54 & 23 \\
\hline Outros & 44 & 19 & 44 & 19 & 36 & 16 \\
\hline \multicolumn{7}{|c|}{ Grau de conhecimento da saúde } \\
\hline Muito bom & 59 & 25 & 71 & 30 & 42 & 18 \\
\hline Bom & 76 & 32 & 79 & 33 & 83 & 36 \\
\hline Relativamente bom & 30 & 12 & 43 & 18 & 46 & 20 \\
\hline Pouco & 45 & 19 & 38 & 16 & 42 & 18 \\
\hline Muito pouco & 28 & 12 & 7 & 3 & 18 & 8 \\
\hline
\end{tabular}

Quanto ao grau de parentesco entre os idosos e adultos A1, a maioria era pais/filhos e, entre os adultos A1 e A2, $42 \%$ eram pais/filhos (79\% eram filhos falando dos pais e $21 \%$ pais falando de filhos) e 23\% irmãos (Tabela 1).

A discordância sobre avaliação da saúde diferiu pouco segundo a perspectiva de resposta do informante secundário. Os adultos A2 e idosos discordaram dos adultos Al em 57\% e 59\% dos casos quando informavam sua própria percepção, e em 55\% e 57\% quando respondiam sobre como percebiam que o adulto Al se auto-avaliava, respectivamente. Quando o adulto Al falava como percebia a saúde do idoso, a discordância foi de $57 \%$, um pouco inferior à discordância observada (63\%) quando o informante secundário respondia utilizando a perspectiva do idoso.

No geral, entre os três pares de informantes primário-secundários, os pares idoso-adulto tiveram concordâncias piores. A maior concordância observada foi para a pergunta relativa à avaliação da saúde, e a menor para o relato de consulta médica nos últimos 12 meses. Quando o adulto Al falava da saúde do idoso e quando este falava da saúde do adulto A1, a avaliação da saúde foi a única variável com concordância observada maior que $90 \%$ (Tabela 2). Quando o adulto A2 falava da saúde do adulto A1, a concordância observada foi maior que $90 \%$ para tabagismo e avaliação da saúde (Tabela 3). Os informantes secundários do adulto Al tiveram concordâncias observadas semelhantes, exceto sobre consulta médica, na qual os adultos A2 concordaram mais (Tabelas 2 e 3 ).

As variáveis que tiveram concordância medida pelo kappa satisfatória foram tabagismo e relato de hipertensão. Os menores índices de kappa foram observados para a avaliação da saúde, 
Tabela 2

Análise da confiabilidade entre informantes primário (adulto A1) e secundário (idoso) segundo variáveis de modo de vida, avaliação da saúde, morbidade referida e uso dos serviços de saúde.

\begin{tabular}{|c|c|c|c|c|c|c|c|c|}
\hline Subconjunto de questões & Variável & $\begin{array}{l}\text { Concordância } \\
\text { observada (\%) }\end{array}$ & Kappa & IC95\% & $\begin{array}{c}\text { Kappa } \\
\text { ajustado } \\
(2 \mathrm{Po}-1)\end{array}$ & $P_{p x}(\%)$ & $P_{p r}(\%)$ & VP (\%) \\
\hline Modo de vida & $\begin{array}{c}\text { Tabagismo } \\
(0=\text { não/1 = sim })\end{array}$ & 89 & 0,76 & $0,68-0,85$ & 0,78 & 38 & 32 & 0,16 \\
\hline \multirow[t]{2}{*}{ Avaliação da saúde } & $\begin{array}{l}\text { Avaliação da saúde } \\
\text { A (muito boa, boa, } \\
\text { regular, ruim, muito } \\
\text { ruim) }\end{array}$ & 93 & $0,33^{*}$ & $0,23-0,38$ & 0,86 & 11 & 10 & 0,09 \\
\hline & $\begin{array}{l}\text { Avaliação da saúde } \\
\text { B (muito boa, boa, } \\
\text { regular, ruim, muito } \\
\text { ruim) }\end{array}$ & 93 & $0,28^{*}$ & $0,14-0,31$ & 0,86 & 11 & 11 & 0 \\
\hline Morbidade referida & $\begin{array}{c}\text { Hipertensão } \\
(0=\text { não/1 = sim })\end{array}$ & 84 & 0,63 & $0,52-0,73$ & 0,68 & 33 & 31 & 0,06 \\
\hline Uso dos serviços de saúde & $\begin{array}{l}\text { Consulta médica } \\
\text { últimos } 12 \text { meses } \\
\text { ( } 0=\operatorname{sim} / 1=\text { não) }\end{array}$ & 82 & 0,49 & $0,36-0,62$ & 0,64 & 21 & 26 & $-0,24$ \\
\hline
\end{tabular}

IC95\%: intervalo de 95\% de confiança; $P$ o: concordância observada; $P_{p x}$ : proporção de respostas positivas dadas pelo informante primário; $P_{\text {pr }}$ proporção de respostas positivas dadas pelo proxy; VP (viés proporcional) $=\left(P_{p x}-P_{p r}\right) / P_{p x}$.

Avaliação da saúde $A$ = como o informante secundário vê a saúde do informante primário (perspectiva do proxy).

Avaliação da saúde $B$ = como o informante secundário acha que o informante primário vê sua própria saúde (perspectiva do informante primário).

Nota: os totais podem diferir um pouco devido a perdas.

* Kappa ponderado usando os escores: $1 ; 0,9375 ; 0,75 ; 0,4375$ e 0.

sendo que houve maior reprodutibilidade quando o adulto A2 falava da saúde do adulto A1 do que quando o adulto A1 falava da saúde do idoso. $\mathrm{Na}$ maioria das variáveis investigadas, o informante secundário do idoso concordou mais com o idoso quando este apresentava o fator pesquisado, o contrário dos informantes secundários dos adultos, que concordaram mais com o adulto Al quando estes não apresentavam o fator investigado (Tabelas 3 e 4).

Os índices do kappa ajustado pela prevalência foram melhores que o kappa simples, essencialmente quando a concordância observada era elevada e o evento era raro, como na avaliação da saúde e consulta médica nos últimos 12 meses. Todas as questões investigadas tiveram concordâncias satisfatórias, medidas pelo kappa ajustado, com exceção de consulta médica quando o idoso falava da saúde do adulto A1 (Tabela 3).

A diferença proporcional entre a prevalência estimada (viés proporcional) com base nos dados dos informantes primários e secundários está apresentada nas Tabela 2, 3 e 4. As estimativas obtidas pelo informante secundário foram quase sempre superestimadas quando o adulto Al falava da saúde do idoso, sendo a maior discrepância observada sobre avaliação da saúde da perspectiva do informante primário (Tabela 4). Quando o informante secundário era o adulto A2, três variáveis foram subestimadas e duas superestimadas (Tabela 3); quando o idoso falava da saúde do adulto Al, três variáveis foram subestimadas, uma superestimada e uma não apresentou viés (Tabela 2).

As probabilidades marginais foram similares na maioria dos eventos investigados entre todos os pares de informantes primário-secundários, tendo diferido mais que quatro pontos quando o adulto Al falava do idoso sobre a avaliação da saúde na perspectiva do idoso e sobre consulta médica.

$\mathrm{Na}$ análise de regressão univariada sobre fatores associados à discordância do adulto Al quando falava da saúde do idoso, somente consulta médica nos últimos 12 meses esteve associada estatisticamente à escolaridade dos informantes secundários. Informantes secundários com oito anos ou mais de estudo tiveram maior 
Análise da confiabilidade entre informantes primário (adulto A1) e secundário (adulto A2) segundo variáveis de modo de vida, avaliação da saúde, morbidade referida e uso dos serviços de saúde.

\begin{tabular}{|c|c|c|c|c|c|c|c|c|}
\hline Subconjunto de questões & Variável & $\begin{array}{l}\text { Concordância } \\
\text { observada (\%) }\end{array}$ & Kappa & IC95\% & $\begin{array}{c}\text { Kappa } \\
\text { ajustado } \\
(2 P o-1)\end{array}$ & $P_{p x}(\%)$ & $P_{p r}(\%)$ & VP (\%) \\
\hline Modo de vida & $\begin{array}{c}\text { Tabagismo } \\
(0=\text { não/1 = sim })\end{array}$ & 92 & 0,84 & $0,76-0,91$ & 0,84 & 39 & 38 & 0,03 \\
\hline \multirow[t]{2}{*}{ Avaliação da saúde } & $\begin{array}{l}\text { Avaliação da saúde } \\
\text { A (muito boa, boa, } \\
\text { regular, ruim, muito } \\
\text { ruim) }\end{array}$ & 93 & 0,37 & $0,32-0,42$ & 0,86 & 12 & 11 & 0,08 \\
\hline & $\begin{array}{l}\text { Avaliação da saúde } \\
\text { B (muito boa, boa, } \\
\text { regular, ruim, muito } \\
\text { ruim) }\end{array}$ & 94 & 0,42 & $0,40-0,48$ & 0,88 & 12 & 10 & 0,17 \\
\hline Morbidade referida & $\begin{array}{c}\text { Hipertensão } \\
(0=\text { não/1 = sim })\end{array}$ & 85 & 0,68 & $0,59-0,78$ & 0,70 & 34 & 38 & $-0,12$ \\
\hline Uso dos serviços de saúde & $\begin{array}{l}\text { Consulta médica } \\
\text { últimos } 12 \text { meses } \\
\text { (0= sim/1 = não) }\end{array}$ & 77 & 0,40 & $0,26-0,53$ & 0,54 & 22 & 28 & $-0,27$ \\
\hline
\end{tabular}

IC95\%: intervalo de 95\% de confiança; $P$ o: concordância observada; $P_{p x}$ : proporção de respostas positivas dadas pelo informante primário; $P_{\text {pr: }}$ proporção de respostas positivas dadas pelo proxy; VP (viés proporcional) $=\left(P_{p x}-P_{p r}\right) / P_{p x}$.

Avaliação da saúde $A$ = como o informante secundário vê a saúde do informante primário (perspectiva do proxy).

Avaliação da saúde $B$ = como o informante secundário acha que o informante primário vê sua própria saúde (perspectiva do informante primário)

Nota: os totais podem diferir um pouco devido a perdas.

* Kappa ponderado usando os escores: $1 ; 0,9375 ; 0,75 ; 0,4375$ e 0.

discordância sobre consulta médica nos últimos 12 meses quando comparados aos com até três anos de estudo (Tabela 5).

Quando o adulto A2 era o informante secundário do adulto $\mathrm{A} 1$, somente a discordância sobre relato de hipertensão esteve associada estatisticamente à idade e escolaridade do adulto A2. Houve maior discordância quando o adulto A2 tinha 60 anos ou mais de idade em relação àqueles com 18 a 39 anos e, quando tinha oito anos ou mais de estudo quando comparado aos com até três anos de estudo (Tabela 5).

Quando o idoso foi o informante secundário do adulto $\mathrm{Al}$, somente relato de hipertensão esteve associado ao grau de conhecimento da saúde do informante primário pelo idoso (Tabela 5).

\section{Discussão}

De forma geral, este estudo verificou boa concordância e reprodutibilidade entre as informações coletadas do indivíduo e de seu informante secundário em relação ao tabagismo, avaliação da saúde, morbidade referida e consulta médica, sendo encontrada pior concordância e confiabilidade entre os pares idoso-adulto, na maioria das variáveis investigadas.

As diferenças encontradas nas porcentagens de concordâncias observadas entre os pares de informantes sofreram influência da idade e escolaridade do informante secundário, além do grau de conhecimento deste sobre a saúde do informante primário. A idade e a escolaridade estiveram inversamente associadas a discordâncias com relação ao relato de diagnóstico médico de hipertensão, sendo que a escolaridade também foi associada a discordâncias sobre consultas médicas.

A concordância entre os pares indivíduoinformante secundário não esteve associada ao sexo do informante secundário, replicando observação anterior relacionada à saúde em idosos 7; porém, diferente do relatado em estudo sobre consumo de álcool 21 e sobre transtornos mentais 19 , em que as mulheres produziram informações mais confiáveis que os homens. Entretanto, uma pesquisa encontrou maior 
Tabela 4

Análise da confiabilidade entre informantes primário (idoso) e secundário (adulto A1) segundo variáveis de modo de vida, avaliação da saúde, morbidade referida e uso dos serviços de saúde.

\begin{tabular}{|c|c|c|c|c|c|c|c|c|}
\hline Subconjunto de questões & Variável & $\begin{array}{l}\text { Concordância } \\
\text { observada (\%) }\end{array}$ & Kappa & IC95\% & $\begin{array}{c}\text { Kappa } \\
\text { ajustado } \\
(2 P o-1)\end{array}$ & $P_{p x}(\%)$ & $P_{p r}(\%)$ & VP (\%) \\
\hline Modo de vida & $\begin{array}{c}\text { Tabagismo } \\
(0=\text { não/1 = sim })\end{array}$ & 85 & 0,71 & $0,62-0,80$ & 0,70 & 46 & 47 & $-0,02$ \\
\hline \multirow[t]{2}{*}{ Avaliação da saúde } & $\begin{array}{l}\text { Avaliação da saúde } \\
\text { A (muito boa, boa, } \\
\text { regular, ruim, muito } \\
\text { ruim) }\end{array}$ & 92 & 0,26 * & $0,15-0,30$ & 0,84 & 15 & 21 & 0,40 \\
\hline & $\begin{array}{l}\text { Avaliação da saúde } \\
\text { B (muito boa, boa, } \\
\text { regular, ruim, muito } \\
\text { ruim) }\end{array}$ & 92 & 0,28 * & $0,24-0,29$ & 0,84 & 15 & 24 & 0,60 \\
\hline Morbidade referida & $\begin{array}{c}\text { Hipertensão } \\
(0=\text { não/1 = sim })\end{array}$ & 85 & 0,60 & $0,49-0,72$ & 0,70 & 73 & 74 & 0,01 \\
\hline Uso dos serviços de saúde & $\begin{array}{l}\text { Consulta médica } \\
\text { últimos } 12 \text { meses } \\
\text { (0= sim/1 = não) }\end{array}$ & 82 & 0,40 & $0,26-0,55$ & 0,64 & 22 & 15 & $-0,32$ \\
\hline
\end{tabular}

IC95\%: intervalo de 95\% de confiança; $P$ o: concordância observada; $P_{p x}$ : proporção de respostas positivas dadas pelo informante primário; $P_{\text {pr: }}$ proporção de respostas positivas dadas pelo proxy; VP (viés proporcional) $=\left(P_{p x}-P_{p r}\right) / P_{p x}$.

Avaliação da saúde $A$ = como o informante secundário vê a saúde do informante primário (perspectiva do proxy).

Avaliação da saúde B = como o informante secundário acha que o informante primário vê sua própria saúde (perspectiva do informante primário)

Nota: os totais podem diferir um pouco devido a perdas.

* Kappa ponderado usando os escores: $1 ; 0,9375 ; 0,75 ; 0,4375$ e 0.

concordância sobre atividades da vida diária quando o informante secundário era do sexo masculino 22 .

Em relação à idade, os adultos A2 com 60 anos ou mais discordaram estatisticamente do informante primário com relação à hipertensão referida. Esse resultado diverge de estudo anterior que analisou a concordância entre idosas e informantes secundários sobre medidas de saúde e estados funcionais, no qual os informantes secundários com 60 anos ou mais se aproximaram mais dos informantes primários 2 .

A associação encontrada entre a maior escolaridade e a pior concordância sobre consulta médica e sobre hipertensão quando os informantes secundários eram o adulto $\mathrm{A} 1 \mathrm{e}$ o adulto A2, respectivamente, pode ser explicada pelo fato de as pessoas com maior escolaridade passarem mais tempo fora de casa por motivo de trabalho (OR de Mantel Haenszel entre trabalho e escolaridade $=2,42$; IC95\%: 1,33-4,41 - dados não mostrados). Pesquisa sobre a concordância de informações entre adolescentes e suas mães também encontrou associação inversa entre escolaridade e grau de concordância quando investigou sintomas depressivos 23 .

A avaliação dos informantes secundários sobre seus conhecimentos acerca da saúde do informante primário foi importante para explicar parte da discordância observada sobre diagnóstico de hipertensão quando os adultos A2 informaram conhecer pouco ou muito pouco a saúde do adulto A1. Resultados de estudo que comparou respostas sobre problemas de saúde de idosos e informantes secundários sugerem que a concordância era melhor quando os informantes se consideravam preparados para dar uma resposta definitiva 7 . A introdução da pergunta acerca do quanto o informante secundário conhece sobre a saúde do indivíduo pesquisado em estudos, pode subsidiar uma crítica mais objetiva a respeito da qualidade dos dados obtidos.

A freqüência dos eventos da maioria das variáveis investigadas foi superestimada pelos informantes secundários dos idosos. Essa tendência também foi observada em pesquisa com 50 casais sobre o relato recente de sintomas neuróticos, psicossomáticos e vagais 24 e em dois 
Características do informante secundário associadas estatisticamente à discordância de informações do informante primário em relação à presença de fatores selecionados.

\begin{tabular}{|c|c|c|c|}
\hline \multicolumn{4}{|c|}{ Características do adulto A1 (informante secundário) associadas estatisticamente à discordância do idoso (informante primário) } \\
\hline Variáveis discordantes & Características do informante secundário & RP & IC95\% \\
\hline Consulta médica nos últimos 12 meses & Escolaridade (em anos) & & \\
\hline \multirow[t]{3}{*}{ Pares discordantes $=36(15 \%)$} & $0-3$ & 1,00 & \\
\hline & 4-7 & 1,98 & $0,91-4,29$ \\
\hline & $8-20$ & 3,03 & $1,37-6,75$ \\
\hline
\end{tabular}

Características do adulto A2 (informante secundário) associadas estatisticamente à discordância do adulto A1 (informante primário)

\begin{tabular}{|c|c|c|c|}
\hline Variáveis discordantes & Características do informante secundário & RP & IC95\% \\
\hline Hipertensão & Idade (em anos) & & \\
\hline \multirow[t]{7}{*}{ Pares discordantes = 34 (15\%) } & $18-39$ & 1,00 & \\
\hline & $40-59$ & 1,00 & $0,44-2,28$ \\
\hline & $60-79$ & 3,62 & $1,87-6,99$ \\
\hline & Escolaridade (em anos) & & \\
\hline & $0-3$ & 1,00 & \\
\hline & $4-7$ & 0,69 & $0,27-1,79$ \\
\hline & $8-20$ & 2,08 & $1,06-4,09$ \\
\hline
\end{tabular}

Características do idoso (informante secundário) associadas estatisticamente à discordância do adulto A1 (informante primário)

\begin{tabular}{|c|c|c|c|}
\hline Variáveis discordantes & Características do informante secundário & RP & IC95\% \\
\hline Hipertensão & Grau de conhecimento da saúde & & \\
\hline \multirow[t]{3}{*}{ Pares discordantes = $39(16 \%)$} & Muito bom/Bom & 1,00 & \\
\hline & Relativamente bom & 0,50 & $0,12-2,05$ \\
\hline & Pouco/Muito pouco & 1,95 & $1,09-3,48$ \\
\hline
\end{tabular}

RP: razão de prevalência; IC95\%: intervalo de 95\% de confiança; Evento: pares discordantes.

estudos que tinham idosos como informantes primários 22,25. Entretanto, quando o informante primário era o adulto $\mathrm{Al}$, três entre as quatro variáveis investigadas foram subestimadas. Estudo que analisou a confiabilidade de casais $(67 \%$ até 35 anos de idade), mostrou que o informante secundário tende a subinformar a presença de sintomas relacionados a transtornos mentais do seu cônjuge 19, fato também observado no National Population Health Survey ${ }^{3}$ no Canadá, em 1996 e 1997. Quanto à natureza dos eventos investigados, os resultados encontrados indicam que o informante secundário ideal pode diferir conforme a natureza da questão investigada. Observou-se melhor confiabilidade entre as variáveis mais objetivas, estáveis e de duração prolongada como tabagismo e hipertensão, em comparação com eventos mais instáveis como consultas médicas. Esse resultado é coerente com o de outros estudos 4,7,18,22,25,26.
Com relação à perspectiva de resposta adotada pelo informante secundário ao responder sobre a saúde do informante primário, os resultados indicam que os adultos Al discordaram menos sobre avaliação da saúde dos idosos quando utilizavam a perspectiva do informante primário.

Apesar da ausência de erros sistemáticos na mensuração das variáveis investigadas, não é possível descartar a presença de erros não diferenciais de classificação de algumas variáveis, como a escolaridade, além de um possível confundimento residual devido à limitação das variáveis consideradas na modelagem.

A generalização dos dados deve ser vista com cautela para populações com características diferentes das estudadas. No caso do kappa, por exemplo, o próprio estimador pode ser afetado pela prevalência do fenômeno estudado, o que torna muitas vezes inapropriado comparar o 
kappa obtido em diferentes estudos ou populações 27.

É importante salientar que a confiabilidade estimada pelo kappa pode não ser uma medida confiável para eventos raros, visto que é afetada pela prevalência 14 . Nesses casos, baixos valores de kappa não refletem necessariamente baixas taxas de concordância total. Para lidar com esse paradoxo, observado principalmente em relação à avaliação da saúde, consulta médica e internação, utilizou-se o índice do kappa ajustado.

A avaliação das probabilidades marginais também demonstrou que o kappa não ajustado é um bom estimador para confiabilidade quando as mesmas são similares entre os pares de informantes primário-secundários.

Outro aspecto a ser considerado no uso do kappa é a adoção de pesos arbitrários atribuídos pelo kappa ponderado para valorizar discordâncias entre categorias próximas como "muito boa" e "boa" e desprezar discordâncias entre categorias distantes como "muito ruim" e "muito boa".

Por fim, salienta-se que os dados gerados em entrevistas de informantes secundários são sempre uma aproximação da informação prestada pelo informante primário, conforme implícito na noção de substituição, proxy ou informação secundária. Nesse sentido, o presente estudo, ao investigar a confiabilidade da informação substituta por meio de medidas de confiabilidade, buscou identificar padrões de aproximação consistentes ou não a fim de verificar em que medida estudos baseados no auto-relato, que usam o informante secundário, obtêm informações iguais às prestadas pelo informante primário.

\section{Conclusão}

Apesar da boa concordância e confiabilidade das informações obtidas entre o auto-relato e o informante secundário na maioria das variáveis investigadas, os resultados indicam que o uso do informante secundário pode introduzir viés na mensuração de exposições e eventos em saúde conforme a natureza da questão investigada e características do informante secundário como a escolaridade e a idade. Além disso, a concordância também reflete o quanto o informante secundário julga conhecer sobre a saúde do informante primário.

Nossos resultados reforçam que a avaliação geral do estado de saúde deve ser uma variável mensurada apenas pelo próprio sujeito devido a sua subjetividade. A mensuração de estados de saúde intermediários (bom, relativamente bom e ruim) é mais passível de discrepância entre os informantes primário-secundários do que estados de saúde nas categorias mais extremas, muito bom ou muito ruim 16. O uso do informante secundário deve se restringir aos casos em que o informante primário é muito jovem, apresenta comprometimento cognitivo, está muito debilitado ou possui uma disfunção que o impeça de responder.

A influência da perspectiva adotada pelo informante secundário em sua resposta deve ser melhor investigada. Isso seria possível se inquéritos de saúde explicitassem qual o ponto de vista utilizado pelo informante secundário em suas respostas.

Acreditamos que a inclusão de uma pergunta nos inquéritos que utilizam o informante secundário sobre o quanto este informante acredita conhecer sobre a saúde do informante primário pode contribuir para uma crítica mais embasada do uso de dados obtidos de informante secundário em estudos seccionais. 


\section{Resumo}

O uso de informante secundário, recurso utilizado no Brasil, pela Pesquisa Nacional por Amostra de Domicílios, pode ser uma fonte de viés em estudos epidemiológicos. Este estudo objetiva estimar a concordância, confiabilidade, magnitude e direção de vieses, e a influência de algumas co-variáveis na discordância entre informações de 710 pares de informantes primário-secundário. A influência do ponto de vista adotado pelo informante secundário, se própria ou na perspectiva do informante primário, também foi investigada. As variáveis pesquisadas estão relacionadas ao modo de vida, avaliação da saúde, morbidade referida e uso de serviços de saúde. Os resultados mostram, em geral, boa concordância. As características dos informantes secundários associadas estatisticamente à discordância foram: idade mais velha, menores escolaridade e conhecimento sobre a saúde do informante primário. Os adultos discordaram menos sobre a avaliação da saúde dos idosos quando utilizaram a perspectiva dos idosos para responder. Os resultados confirmam a presença de viés ao utilizar o informante secundário para hipertensão e consulta médica.

Viés (Epidemiologia); Saúde do Idoso; Avaliação em Saúde; Reprodutibilidade dos Resultados

\section{Referências}

1. Armstrong BK, White E, Saracci R. Principles of exposure measurement in epidemiology. New York: Oxford University Press; 1992.

2. Nelson LM, Longstreth Jr. WT, Koepsell TT, van Belle G. Proxy respondents in epidemiologic research. Epidemiol Rev 1990; 12:71-86.

3. Shields M. Proxy reporting in the National Population Health Survey. Health Rep 2000; 12:21-39.

4. Milne DJ, Mulder LL, Beelen HCM, Schoefield P, Kempen GIJM, Aranda S. Patients' self-reporting and family caregivers' perception of quality of life in patients with advanced cancer: how do they compare? Eur J Cancer Care (Engl) 2006; 15: 125-32.

5. Zanei SSV. Análise dos instrumentos de avaliação de qualidade de vida WHOQOL-bref e SF-36: confiabilidade, validade e concordância entre pacientes de Unidades de Terapia Intensiva e seus familiares [Tese de Doutorado]. São Paulo: Escola de Enfermagem, Universidade de São Paulo; 2006.

\section{Colaboradores}

R. Jardim contribuiu na coleta de dados, análise e redação do artigo. S. M. Barreto colaborou na análise, revisão e redação do artigo. L. Giatti participou na análise e revisão do artigo.

\section{Agradecimentos}

A todos os participantes do estudo, funcionários da Vila Pinho, bolsistas e demais pessoas envolvidas no projeto, à Fundação de Amparo à Pesquisa de Minas Gerais (FAPEMIG) e ao Fundo Nacional de Saúde (FNS) pelo financiamento. S. M. Barreto é bolsista de produtividade em pesquisa do Conselho Nacional de Desenvolvimento Científico e Tecnológico (CNPq) e L. Giatti é bolsista do Programa de Apoio a Projetos Institucionais com a Participação de Recém-Doutores (PRODOC) da Coordenação de Aperfeiçoamento de Pessoal de Nível Superior (CAPES).
6. von Essen L. Proxy ratings of patient quality of life - factors related to patient-proxy agreement. Acta Oncol 2004; 43:229-34

7. Dewey CJP; Analysis Group of the MRC-FEA Study. Survey into health problems of elderly people: a comparison of self-report with proxy information. Int J Epidemiol 2000; 29:684-97.

8. McPhail S, Beller E, Haines M, Haines T. Two perspectives of proxy reporting of health-related quality of life using the Euroqol-5D, an investigation of agreement. Med Care 2008; 46:1140-8.

9. Malta DC, Leal MC, Lima-Costa MF, Morais Neto OL. Inquéritos nacionais de saúde: experiência acumulada e proposta para o inquérito de saúde brasileiro. Rev Bras Epidemiol 2008; 11 Suppl 1:S159-67.

10. Barros MBA, César CLG, Carandina L, Torre GD. Desigualdades sociais na prevalência de doenças crônicas no Brasil, PNAD-2003. Ciênc Saúde Coletiva 2006; 11:911-26. 
11. Almeida MF, Barata RB, Montero CV, Silva ZP. Prevalência de doenças crônicas auto-referidas e utilização de serviços de saúde, PNAD/1998, Brasil. Ciênc Saúde Coletiva 2002; 7:743-56.

12. Torres HG, Bichir RM, Carpim TP. Uma pobreza diferente? Mudanças no padrão de consumo da população de baixa renda. Novos Estudos CEBRAP 2006; 74:17-22.

13. Lima-Costa MF, Barreto SM, Giatti L. Condições de saúde, capacidade funcional, uso de serviços de saúde e gastos com medicamentos da população idosa brasileira: um estudo descritivo baseado na Pesquisa Nacional por Amostra de Domicílios. Cad Saúde Pública 2003; 19:735-43.

14. Carvalho AC. Fatores associados ao desempenho na compreensão da linguagem oral em idosos: projeto envelhecimento em saúde [Dissertação de Mestrado]. Belo Horizonte: Faculdade de Medicina, Universidade Federal de Minas Gerais; 2008.

15. Hyland A, Cummings KM, Lynn WR, Corle D, Giffen CA. Effect of proxy -reported smoking status on population estimates of smoking prevalence. Am J Epidemiol 1997; 145:746-51.

16. Sneeuw KCA, Sprangers MAG, Aaronson NK. The role of health care providers and significant others in evaluating the quality of life of patients with chronic disease. J Clin Epidemiol 2002; 55: 1130-43.

17. Rao CR, Miller JP, Rao DC. Handbook of statistics, 27. Epidemiology and medical statistics. Amsterdam: Elsevier; 2007.

18. Slater MB. Concurrent assessment of interrater agreement and intrarater reliability in the case of binay data [Dissertação de Mestrado]. Calgary: University of Calgary; 2006.
19. Santana VS, Almeida Filho N, Rocha CO, Matos AS. Confiabilidade e viés do informante secundário na pesquisa epidemiológica: análise de questionário para triagem de transtornos mentais. Rev Saúde Pública 1997; 31:556-65.

20. Barros AJ, Hirakata VN. Alternatives for logistic regression in cross-sectional studies: an empirical comparison of models that directly estimate the prevalence ratio. BMC Med Res Methodol 2003; 3:21.

21. Graham P, Jackson R. Primary versus proxy respondents: comparability of questionaire data on alcohol consumption. Am J Epidemiol 1993; 138: 443-52.

22. Magaziner J, Basset SS, Hebel JR, Gruber-Baldini AL. Use of proxies to measure health and functional status in epidemiologic studies of communitydwelling women aged 65 years and older. Am J Epidemiol 1996; 143:283-92.

23. Fraga-Maia H, Santana VS. Concordância de informações de adolescentes e suas mães em inquérito de saúde. Rev Saúde Pública 2005; 39:430-7.

24. Briscoe ME. Research note: proxy responses in health surveys: a methodological issue. Sociol Health Illn 1984; 6:359-66.

25. Magaziner J, Zimmerman SI, Gruber-Baldini AL, Hebel JR, Fox KM. Proxy reporting in five areas of functional status. Am J Epidemiol 1997; 146: 418-28.

26. Pickard AS, Knight SJ. Proxy evaluation of healthrelated quality or life - a conceptual framework for understanding multiple proxy perspectives. Med Care 2005; 43:493-9.

27. Dachs JNW, Santos APR. Auto-avaliação do estado de saúde no Brasil: análise dos dados da PNAD/2003. Ciênc Saúde Coletiva 2006; 11: 887-94.

Recebido em 29/Mar/2009

Versão final reapresentada em 10/Nov/2009

Aprovado em 10/Jun/2010 